\title{
Current Activities in the Interactive Joint Research at Tohoku University - Advanced Evaluation of Radiation Effects on Fusion Materials -*)
}

\author{
Hiroaki KURISHITA, Masahiko HATAKEYAMA, Minoru NARUI, Satoru MATSUO, \\ Tatsuo SHIKAMA, Kohei TADA, Noriyasu OHNO ${ }^{1)}$, Takao TAKEUCHI ${ }^{2)}$, Yuji HATANO ${ }^{3)}$, \\ Masaki NISHIURA $^{4)}$, Yousuke NAKASHIMA ${ }^{5)}$, Hideo WATANABE ${ }^{6)}$, Kazutoshi TOKUNAGA ${ }^{6}$, \\ Tomoaki HINO ${ }^{7)}$, Yoshio UEDA ${ }^{8)}$, Takeo MUROGA ${ }^{4)}$, Akio SAGARA ${ }^{4)}$ and Osamu KANEKO ${ }^{4}$ \\ International Research Center for Nuclear Materials Science, Institute for Materials Research, Tohoku University, \\ Ibaragi 311-1313, Japan \\ ${ }^{1)}$ Graduate School of Engineering, Nagoya University, Nagoya 464-8603, Japan \\ ${ }^{2)}$ National Institute for Materials Science, Tsukuba 305-0047, Japan \\ ${ }^{3)}$ Hydrogen Isotope Research Center, University of Toyama, Toyama 930-8555, Japan \\ ${ }^{4)}$ National Institute for Fusion Science, Toki 509-5292, Japan \\ 5) Plasma Research Center, University of Tsukuba, Tsukuba 305-8577, Japan \\ 6) Institute for Applied Mechanics, Kyushu University, Kasuga 816-8580, Japan \\ 7) Graduate School of Engineering, Hokkaido University, Sapporo 060-8628, Japan \\ ${ }^{8)}$ Graduate School of Engineering, Osaka University, Suita 565-0871, Japan
}

(Received 26 November 2013 / Accepted 5 March 2014)

\begin{abstract}
International Research Center for Nuclear Materials Science of the Institute for Materials Research, Tohoku University (hereafter the "Center") was founded in 1969 and has been playing a vital role as the joint-use research center in Japan to assess the dynamic and static effects of neutron irradiation on the physical and mechanical properties of a variety of structural and functional materials through the use of nuclear reactors in Japan and overseas. The Center is now also open to researchers overseas. As a new initiative, the Center started an interactive joint research scheme on nuclear fusion reactor engineering with the NIFS in fiscal 2010. The interactive joint research aims at pioneering inter-disciplinary fields that connect neutron reactor engineering with other nuclear fusion sciences, and at conducting activities primarily on the key research subjects through inter-research-center collaboration. For this, a TDS (Thermal Desorption Spectrometer) with an ion gun (IG-TDS) has been installed in the radiation controlled area at the Center. Development of a compact divertor plasma simulator (C-DPS) system that will be integrated with the IG-TDS apparatus is in progress. It is prospected that the Center could play a leading role in international collaborative studies of neutron irradiation effects on plasma material interaction, along with other major research institutes over the world.
\end{abstract}

(c) 2014 The Japan Society of Plasma Science and Nuclear Fusion Research

Keywords: interactive joint research, fusion reactor engineering, neutron irradiation, hydrogen isotope retention, plasma facing material, plasma material interaction, thermal desorption spectrometer, compact divertor plasma simulator

DOI: $10.1585 /$ pfr.9.3405136

\section{Introduction}

The International Research Center for Nuclear Materials Science of Institute for Materials Research (IMR), Tohoku University (hereafter the "Center") was founded in 1969. Since then the Center has been playing a vital role as the research center open to university researchers in Japan to assess the effects of neutron irradiation on the physical and mechanical properties of a variety of structural and functional materials through the use of nuclear

author'se-mail: kurishi@imr.tohoku.ac.jp

*) This article is based on the presentation at the 23rd International Toki Conference (ITC23). reactors such as JMTR, Joyo and JRR-3 in Japan, FFTF and HFIR in USA and BR2 in Belgium. The Center is now also open to researchers overseas. Figure 1 shows the Center located close to JMTR and Joyo reactors at the Oarai Site of JAEA (Japan Atomic Energy Agency).

On the other hand, the feature of the nuclear fusion reactor environments is that hydrogen isotopes and helium are produced and their transport and retention occur under neutron irradiation. It is indispensable to clarify the effects of neutron irradiation on the behavior of hydrogen isotopes and helium in the candidate plasma facing materials (PFMs) such as tungsten (W) to assess the feasibility 


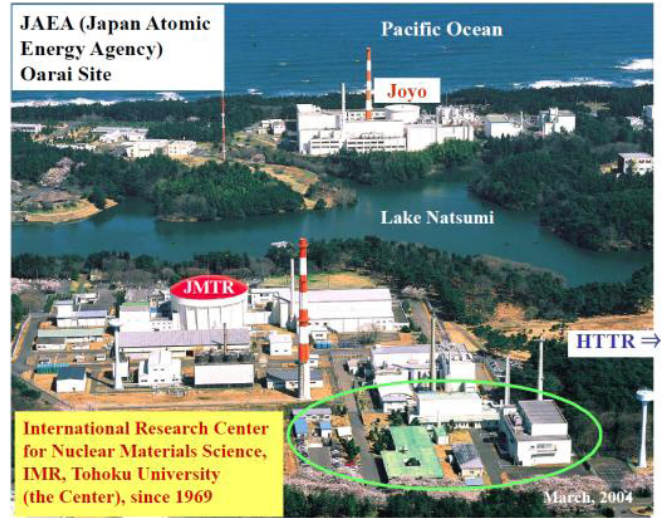

Fig. 1 Bird's-eye view including the Center at Tohoku University and the reactors of JMTR and Joyo at the Oarai Site of JAEA that are indispensable to material irradiation studies.

of their use in fusion reactors.

As a new initiative, therefore, the Center started an interactive joint research scheme on nuclear fusion reactor engineering with NIFS in fiscal 2010. The paper will present current activities in the interactive joint research at the Center with NIFS and other major research institutes.

\section{Concept of Interactive Research Program at the Center}

The Interactive Research Program hosted by the Center aims at the followings.

(1) Framework establishment of new interactive joint projects as the hosting center and rearrangement of ongoing Japan-US cooperative fusion-relevant programs (e.g. PHENIX).

(2) Placement of core research interest in neutron reactor engineering utilizing nuclear reactors and exploitation of inter-disciplinary research fields across related nuclear fusion science and engineering: Plasma irradiation on neutron irradiated materials to be proposed by Tsukuba University (GAMMA 10), Kyushu University (QUEST) and progress of development of Compact Divertor Plasma Simulator (C-DPS) at Nagoya university that will be installed and equipped with TDS (Thermal Desorption Spectrometer) in the radiation controlled area at the Center.

(3) Extensive activities on core research projects with emphasis on inter-institutional cooperation.

(4) Active involvement in confronted important subjects including irradiation effects on hydrogen isotopes in fusion reactor materials and cryogenic irradiation; critical to fusion reactor engineering.

As a first step to realize this concept, a TDS with an ion gun (IG-TDS) has been installed in the radiation controlled area at the Center (Fig. 2).

In the interactive research program at the Center in fiscal 2013, nine research proposals were accepted after

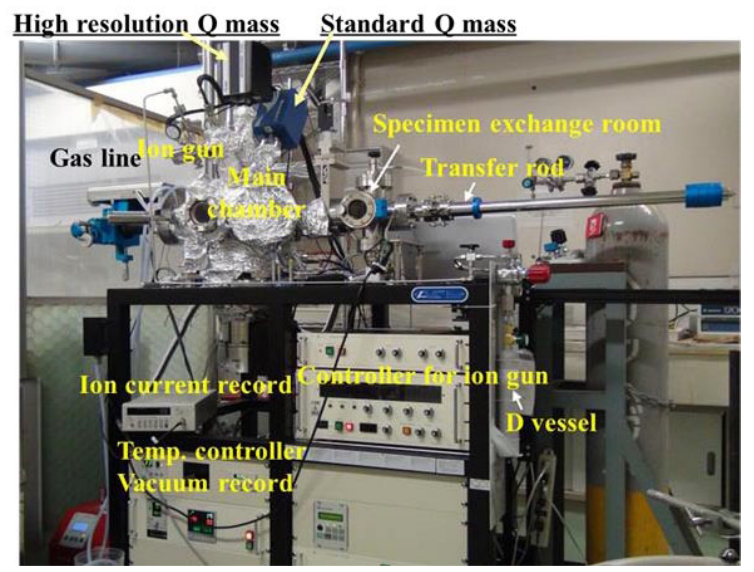

Fig. 2 TDS device equipped with an ion gun (IG-TDS) installed in the radiation controlled area in the Center at Tohoku University.

reviews at NIFS and the Center. Among them "Advanced evaluation of radiation effects on fusion materials" is the underlying project for all of the proposed studies and presented below.

\section{Current Activity on Plasma Ma- terial Interaction (PMI) Study on Candidate PFMs}

\subsection{Current understanding on hydrogen iso- tope retention in tungsten materials}

Critical literature reviews regarding deuterium (D) retention in tungsten materials without synthetic high-energy particle irradiation can be summarized as follows.

(1) The trapping of D at dislocations is dominant and that at grain boundaries is not significant [1].

(2) High D retention is typified by a distinct formation of blisters, showing the importance of blistering phenomena for D inventory.

(3) The formation of blisters or blister-like features results in local increase in the density of dislocations emitted by crack-tip plasticity at the edges of expanded blister cavities. $\mathrm{D}$ is not primarily retained as $\mathrm{D}_{2}$ gas, but is trapped by the dislocations with binding energy of $\sim 1.25 \mathrm{eV}$ [1].

(4) The behavior of the tungsten-hydrogen system is the reflection of a number of interacting effects. Their interplay can even reverse under certain conditions: The dependence of trap density in the initial microstructure on $\mathrm{D}$ inventory is reversed by synergistic displacement damage due to energetic particles [1-3].

On the other hand, PFMs in nuclear fusion reactors will be subjected to concurrent irradiations with highenergy neutrons, low energy ions that cause serious material degradation. The high-energy particle irradiation induces a large number of lattice defects due to displacement damage. The lattice defects act as trapping sites for $\mathrm{D}$ and 


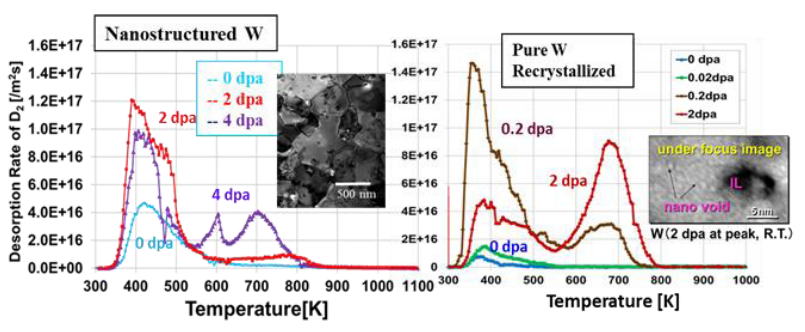

Fig. 3 Effect of displacement damage on desorption rate of $\mathrm{D}_{2}$ for nanostructured $\mathrm{W}$ and pure recrystallized $\mathrm{W}$ before and after $2.4 \mathrm{MeV} \mathrm{Cu}^{2+}$ irradiation. Note that the increase in $\mathrm{D}$ retention by displacement damage is significantly suppressed by the initial microstructure control in $\mathrm{W}$ materials.

significantly increase D retention. Recent studies on the effect of irradiation of high-energy neutrons and heavy ions on $\mathrm{D}$ retention have supported the above view $[4,5]$.

Suppression of D retention requires the prevention of accumulation of radiation induced lattice defects. This will be achieved by using nanostructured $\mathrm{W}$ materials containing a high density of sinks for the defects, such as grain boundaries (GBs) and fine dispersoids.

Figure 3 shows how the effect of displacement damage on $\mathrm{D}$ retention in $\mathrm{W}$ materials is affected by their initial microstructures prior to damaging [6]. Here, $0.1 \mathrm{~mm}$ thick sheets with $3 \mathrm{~mm}$ in diameter of pure $\mathrm{W}$ recrystallized at $2273 \mathrm{~K}(\mathrm{R}-\mathrm{W})$ and nanostructured $\mathrm{W}$ [7] were irradiated with $2.4 \mathrm{MeV}-\mathrm{Cu}^{2+}$ at room temperature up to $2 \times 10^{19}$ ions $/ \mathrm{m}^{2}$, which corresponds to $4 \mathrm{dpa}$ (displacement per atom) with the peak damaged zone around 400$500 \mathrm{~nm}$ from the surface. The damaged specimens were irradiated with $2.0 \mathrm{keV}-\mathrm{D}^{2+}$ at room temperature and subjected to TDS with a heating rate of $1 \mathrm{~K} / \mathrm{s}$. It is obvious from the figure that before $\mathrm{Cu}$ ion irradiation $\mathrm{D}$ retention is much higher in nanostructured $\mathrm{W}$ than in R-W. However, after the $2.4 \mathrm{MeV} \mathrm{Cu}^{2+}$ irradiation, $\mathrm{R}-\mathrm{W}$ exhibits a sharp increase in $\mathrm{D}$ retention, whereas nanostructured $\mathrm{W}$ exhibits D retention less sensitive to the irradiation. This is quite likely due to the differences in the density of sinks for radiation induced defects and thus resultant lattice defects due to $2.4 \mathrm{MeV} \mathrm{Cu}^{2+}$ irradiation between $\mathrm{R}-\mathrm{W}$ and nanostructured $\mathrm{W}$ : As the damage level increases, a distinct peak around $700 \mathrm{~K}$ appears at $0.2 \mathrm{dpa}$ for $\mathrm{R}-\mathrm{W}$ and 4 dpa for nanostructured W. In R-W with 2 dpa a dislocation loop of the interstitial type (IL) and a high density of nanovoids are observed. In view of higher thermal stability of nanovoids than IL, the peak around $700 \mathrm{~K}$ can be attributed to desorption from the nanovoids.

It should be noted that neutron irradiation causes uniform damage/trap creations (uniform PKA energy) contrary to near surface damage by ion-irradiation and the beneficial effects of nanostructures on $\mathrm{D}$ retention are expected to be greater in the neutron irradiated state than in the heavy ion irradiated state.

\section{Development of a New Compact DPS with TDS}

The systematic study of hydrogen isotope retention in neutron-irradiated PFMs under the SOL (Scrape Off Layer) relevant condition requires the development and installation of a linear plasma irradiation device equipped with IG-TDS in the radiation controlled area.

\subsection{Current activities in the world 4.1.1 United States}

TPE (Tritium Plasma Experiment) in INL can handle neutron-irradiated materials, (TITAN project: JapanUS collaboration program), however, analytical apparatus such as TEM, SEM, 3 dimensional atom probe (3D-AP) and positron annihilation (PA) are not available in the radiation controlled area. The size of the specimens should be larger than $6 \mathrm{~mm}$ in diameter.

In PMTS (Plasma Material Test Stand) at ORNL, design and development of an intense RF plasma source (operational with high ion temperature, and not limited by lifetime and impurity issues of solid material sources) are in progress for the project approved.

\subsubsection{EU}

JULE-PSI (Jülich Linear Experiment for PSI studies in a hot cell) in Jülich, which can handle neutron-irradiated materials, is under construction. The analyses of neutronirradiated/plasma-exposed surfaces of PFMs can be performed on the surfaces only after air exposure following plasma exposure, which may lead to concern for the contamination of plasma-exposed surfaces and resultantly less reproducible results.

\subsubsection{Japan}

It is difficult to build a new radiation-controlled facility with divertor plasma simulator. An alternative strategy for PMI studies in neutron-irradiated PFMs is to develop a new compact divertor plasma simulator (C-DPS) and unite it to the existing IG-TDS device in the radiation controlled area at Tohoku University. The C-DPS with compact and high efficient dc plasma source is under progress at Nagoya University based on the prototype of the C-DPS device [8], and the system of C-DPS integrated with the existing IGTDS device (C-DPS/IG-TDS) will be completed and installed at the Center in 2014.

Figure 4 shows the new C-DPS for a small laboratory to focus on PMI issues, together with the range of ion energy and ion flux density available for the C-DPS. The C-DPS can produce a high plasma density $\sim 10^{19} \mathrm{~m}^{-3}$ at $2-2.5 \mathrm{~kW}$ and $20 \mathrm{mT}$ that is steady state plasma relevant to the divertor plasma condition. This has been achieved by using zigzag-shaped $\mathrm{LaB}_{6}$ cathodes heated by direct Joule current for efficient heating $(1600 \mathrm{~K}$ at $730 \mathrm{~W})$ and introducing discharge gases between the two cathodes for efficient usage of neutral gas for discharge. The C-DPS 

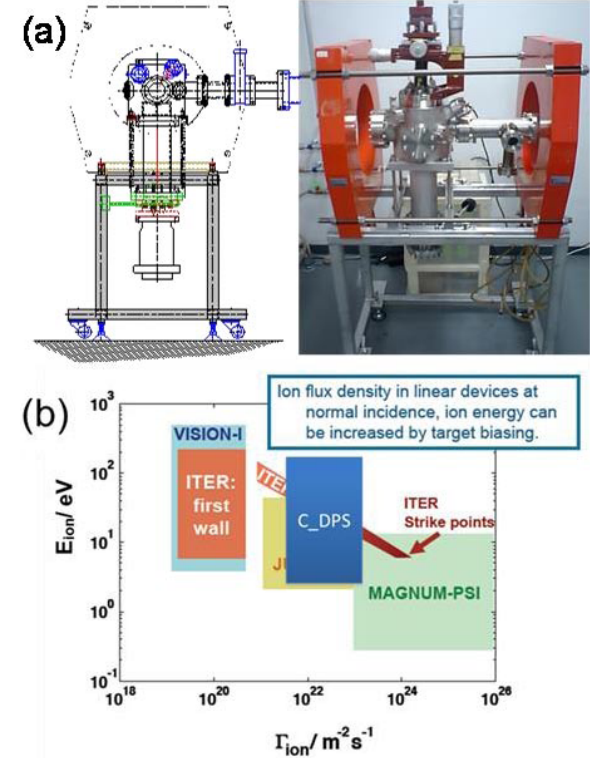

Fig. 4 A new compact divertor plasma simulator (C-DPS) with a high efficient dc plasma source composed of a zigzagshaped $\mathrm{LaB}_{6}$ cathode and a water-cooled hollow cupper anode (a) and ion energy and ion flux density available for the C-DPS (b).

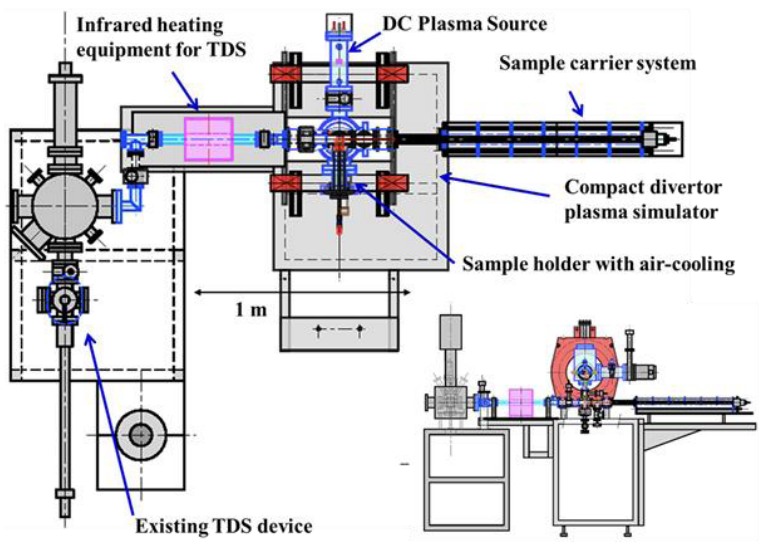

Fig. 5 Design of the system of C-DPS/IG-TDS that enables the TDS analyses of neutron and plasma irradiated surfaces of PFMs without air exposure following plasma irradiation.

has the feature of a compact and easy movable design, low electricity consumption, low gas consumption, and cooling with a water chiller.

Figure 5 shows the design of C-DPS/IG-TDS in the radiation controlled area at Tohoku University. Since the system and the Center have the following attractive features, pioneering results will be expected.

(1) The analyses of neutron and plasma irradiated surfaces of PFMs can be performed without air exposure following plasma irradiation.

(2) Various analytical apparatus such as TEM, SEM, 3D-AP and PA for neutron and plasma irradiated PFMs are available in the radiation controlled area at the Center, and advanced technologies for nanostructure analysis are established.

(3) Many neutron irradiated specimens of PFMs with $3 \mathrm{~mm}$ in diameter and $0.1 \sim 0.2 \mathrm{~mm}$ in thickness are available at the Center.

In Fig. 5, a neutron irradiated sample $(\phi 3.0 \mathrm{~mm} \times \mathrm{t}$ $0.1 \sim 0.2 \mathrm{~mm}$ ) will be mounted on the sample holder. The sample temperature can be controlled by air cooling. After irradiated with plasma, the sample is transferred from the irradiation-chamber to the IG-TDS device and then removed from the sample holder without breaking vacuum in the following ways:

The plasma irradiated sample is dropped from the holder to bucket of TDS, moved to the infrared heating unit for TDS measurement, then moved back to the original position, and finally turned over the bucket and dropped to the bottom of the chamber

This type of an integrated system has never been available throughout the world, and therefore the system of CDPS/IG-TDS will contribute to the advance of PMI studies particularly by enhancement of reliability of the results obtained, and international research collaboration will be intensified.

\section{Summary}

1) Interactive joint research with NIFS and other major research institutes was initiated to aim at pioneering inter-disciplinary fields that connect neutron reactor engineering with other nuclear fusion sciences, and at conducting activities primarily on the key research subjects through inter-research-center collaboration.

2) The Center at Tohoku University has installed a thermal desorption spectrometer (TDS) with an ion gun (IG-TDS) in its radiation controlled area to systematically investigate the effects of high-energy particle irradiation on $\mathrm{D}$ retention in candidate plasma facing materials (PFMs).

3) Development of a new compact divertor plasma simulator (C-DPS) and its incorporation with the existing IG-TDS device in the radiation controlled area at the Center is in progress. Since the system of C-DPS/IG-TDS will enable the analyses of neutron and plasma irradiated surfaces of PFMs without air exposure following plasma irradiation, pioneering results will be expected and international research collaboration will be enhanced.

[1] A. Manhard, PhD thesis at the Max-Planck-Institute for plasma Physics, Garching; at the University of Augsburg, December 2011.

[2] V. Arimov et al., J. Nucl. Mater. 375, 192 (2008).

[3] M. Miyamoto et al., Nucl. Fusion 49, 065035 (2009).

[4] M. Shimada et al., J. Nucl. Mater. 415, S667S671 (2011).

[5] Y. Hatano et al., J. Nucl. Mater. 438, S114 (2013).

[6] H. Kurishita et al., Phys. Scr. T159, 014032 (2014).

[7] H. Kurishita et al., Mater. Trans. 54, 456 (2013).

[8] M. Yamagiwa et al., Phys. Scripta T145, 014032 (2011). 